\title{
Improving the reliability of a Bridge Management System (BMS) using an ANN- based Backward Prediction Model (BPM)
}

\author{
${ }^{1}$ Jaeho Lee, ${ }^{2}$ Kamalarasa Sanmugarasa, ${ }^{3}$ Michael Blumenstein and ${ }^{1}$ Yew-Chaye Loo \\ ${ }^{1}$ Griffith School of Engineering, Gold Coast Campus, Griffith University, QLD 4222, Australia \\ ${ }^{2}$ Parsons Brinckerhoff Australia P/L, GPO Box 2907, Brisbane, QLD 4001, Australia \\ ${ }^{3}$ School of Information and Communication Technology, Gold Coast Campus, Griffith University, \\ QLD 4222, Australia
}

\begin{abstract}
The slow adoption of Bridge Management Systems (BMSs) and its impractical future prediction of the condition rating of bridges are attributed to the inconsistency between BMS inputs and bridge agencies' existing data for a BMS in terms of compatibility and the enormous number of bridge datasets that include historical structural information. Among these, historical bridge element condition ratings are some of the key pieces of information required for bridge asset prioritisation but in most cases only limited data is available.
\end{abstract}

This study addresses the abovementioned difficulties faced by bridge management agencies by using limited historical bridge inspection records to model time series element level data. This paper presents an Artificial Neural Network (ANN) based prediction model, called the Backward Prediction Model (BPM), for generating historical bridge condition ratings using limited bridge inspection records. The BPM employs historical non-bridge datasets such as traffic volumes, populations and climates, to establish correlations with existing bridge condition ratings from very limited bridge inspection records. The resulting model predicts the missing historical condition ratings of individual bridge elements. The outcome of this study can contribute to reducing the uncertainty in predicting future bridge condition ratings and so improve the reliability of various BMS analysis outcomes.

Keywords: Bridge Condition Ratings, Bridge Management System (BMS), Artificial Neural Network (ANN), Backward Prediction Model (BPM)

\subsection{Introduction}

The efficient use of public funds for the well-being of bridge networks requires an effective bridge asset management technology. It is particularly important to optimise future bridge maintenance, repair and rehabilitation (MR\&R) activities with the given funding and to request suitable future funding based on reliable Bridge Management System (BMS) outcomes. A BMS, as a computer-based decision support system (DSS), is used to determine the best possible strategy that ensures an adequate level of safety for bridges at the lowest possible life-cycle cost [1]. Many bridge agencies worldwide have begun the transition to BMS-based bridge asset management. A BMS, based on the results of a deterioration model, provides various important future estimations for the planning of MR\&R activities. The success of a BMS is highly 
dependent on the accurate estimation of future condition ratings [2]. The condition ratings are used directly and indirectly as input data for many significant functions in the commercial BMS package [3]. Fig. 1 presents the uses of bridge condition ratings and the relationship with many analytical BMS modules in project and network level analysis. Ideally, a BMS should identify current and future bridge deficiencies and estimate the backlog of funding requirements. Typical BMS software mainly functions to [3,4]: (1) forecast future bridge deficiencies; (2) identify a list of improvement options to correct such deficiencies; and (3) estimate the costs and benefits of implementing each improvement option.

Fig.1 Re-illustration of relationships between historical bridge inspection data and BMS outputs [4]

(Note that the relationships with other input sources and BMS outputs are omitted)

Numerous bridge condition rating and deterioration models have been developed to reliably determine the bridge life cycle for the remaining years of use and major MR\&R needs. However, the estimations of future structural condition ratings from BMSs are still not practical for use in developing reliable long-term maintenance strategies.

From the perspective of bridge agencies, there are a number of shortcomings related to the use of BMS software. Inter alia these are: (1) Commercial BMS software has been used for less than 15 years and even those bridge agencies which implemented BMSs from an early stage, would have only approximately 6 to 7 biennial inspection records at their disposal. (2) Bridge condition ratings normally do not change much over short time periods. (3) Approximately 60\% of BMS analytical processes rely heavily on periodic bridge inspection results [5]. These factors lead to the inaccuracy in predicting the future structural performance of bridges.

The main difficulty faced by current deterioration modelling techniques is the lack of usable data related to the bridge element's historical behaviour. Based heavily on a few sets of recent structural condition ratings, current modelling techniques cannot be expected to produce reliable outcomes. This in turn leads to an unreliable prediction of future bridge condition ratings. Not withstanding the above, the methodology presented in this paper is an ANN-based Backward Prediction Model (BPM), which reliably generates unavailable historical bridge condition ratings. It aims to improve the accuracy of future structural condition ratings.

\subsection{Time-Series Predictions for Insufficient Datasets}

Time-series predictions are important resources for making decisions in many application domains [6]. Various prediction techniques have been applied to commercial BMS analysis modules. The most frequently used techniques are Regression, Markov models, Bayesian methods, Fuzzy techniques, Genetic Algorithms, Case Based Reasoning and Artificial Neural Network (ANN) models. Specifically, Markov decision processes (MDP) have been used in major state-of-theart BMS software as part of their deterioration modules. To obtain reliable predictions from conventional techniques, the size of missing patterns from an entire dataset must 
be $5 \%$ or less [7]. It should be noted that irregularly sampled datasets cannot be used with conventional prediction methods [8].

This research aims to utilise limited inspection records over a short period to predict large datasets spanning over a much longer time period. As mentioned in Section 1, the short history of the BMS adoption and its lack of usable inspection records causes unreliable long-term bridge performance predictions. Recognising the historical patterns for aging bridges can be a problem when using commonly available time series prediction methods.

For any computational prediction methods, the amount of available datasets is required to be much larger than the target prediction datasets to obtain reliable prediction results. An ANN-based model also requires a large number of training datasets to successfully estimate their correlation. It should be noted that existing ANN-based "data-mining" techniques have been applied in medical, economics, engineering and IT fields. While capable of carrying out similar activities as the BPM, data-mining has had success only in cases where very small proportions of the datasets are unavailable - much smaller than are required to be generated for an effective BMS implementation. To rise above the fundamental limitations in timeseries predictions, the proposed neural network model adopts an alternative type of time-series dataset, which is used to overcome the lack of trends in the existing small number of bridge element condition ratings.

To address the research problem indicated, the BPM is described in this paper employing non-bridge historical data to support the lack of trends in the existing bridge condition ratings to generate the unavailable years of historical condition ratings and so establishing some comprehensive datasets.

\subsection{Outline of the Proposed Model}

The research problem regarding the lack of historical bridge data may be solved by the use of an ANN-based Backward Prediction Model (BPM). A pilot study along these lines only considered bridge condition ratings amongst the BMS historical data required. The BPM predicts entire or selected periods of historical bridge condition ratings to generate unavailable years of datasets. It aims to improve the prediction accuracy of future bridge condition ratings using a deterioration module.

The bridge condition ratings in existing small numbers of condition ratings do not change much during a short period of time. As such, it is also difficult to detect condition rating changes using an ANN-based condition rating prediction model. However, existing bridge condition ratings can be strengthened by non-bridge factors including local climates, number of vehicles and population growth in the area surrounding the bridge. The non-bridge factors are employed to help establish the correlations between the non-bridge factors and the lack of historical data patterns in the existing but inadequate bridge condition rating datasets.

Fig.2 schematically describes the mechanism of the BPM. It illustrates the main function of the ANN technique in establishing the correlation between the existing condition rating datasets (from year $\mathrm{m}$ to year $\mathrm{m}+\mathrm{n}$ ) and the corresponding years' non-bridge factors. The non-bridge factors directly and indirectly affect the variation of the bridge conditions thereby the deterioration rate. The relationships established 
using neural networks are then applied to the non-bridge factors (for year 0 to year $\mathrm{m}$ ) to generate the missing bridge condition ratings (for the same year 0 to year $\mathrm{m}$ ). Thus, the non-bridge factors in conjunction with the ANN technique can produce the historical trends that inform the current condition ratings.

\section{Fig.2 Outline of proposed Backward Prediction Model (BPM)}

The BPM has been tested using two different types of bridge condition rating datasets - the National Bridge Inventory (NBI) and BMS condition rating inputs - for the same bridge provided by the Maryland Department of Transport (DoT), USA. The errors cope within $\pm 10 \%$ for the historical bridge condition ratings that are generated. The magnitude of the prediction error depends on the scale of each Condition State (CS) (e.g. 20\% magnitude of each CS in 5 graded CSs) in element level inspection methods for a BMS. In order to generate acceptable historical condition rating datasets for BMS inputs, the proposed model required 4-5 sets of bridge inspection results. The details of this will be further discussed in Section 3.3.

\subsection{Element Level Bridge Inspections}

The most widely used inspection method for a BMS operation is the element-level type. This evolved from the National Bridge Inventory (NBI). NBI information is submitted annually to Federal Highway Administration (FHWA) by state highway agencies in the U.S. NBI has been used for more than three and a half decades to determine the needs of rehabilitation and replacement, considered nation-wide. However, it was found to be insufficient to establish MR\&R. The element level inspection method has the following advantages which have been reported by FHWA: (1) more precise bridge condition assessment; (2) more quantitative condition data of each element beyond deck, superstructure and substructure per bridge; and (3) sustaining the element level inspection method based BMS [10-12]. Thus, the proposed study employs commonly used bridge condition rating information based on an element level inspection method for updating a dataset of the BMS. In addition, NBI information was used to measure directly the performance of the proposed BPM.

The obtained bridge condition datasets require calibration to fit into the proposed BPM model, due to the typical ANN input environment. The acceptable numerical scale for ANN modelling is from -1 to 1 (or 0 to 1 ). Fig.3 illustrates the scale of NBI and element level-based condition rating information for this particular study.

Fig.3 Scale of the condition ratings used in the BPM model

The Condition Index (CI) in NBI is scaled between 0 to 9 for the NBI\#58 (deck), \#59 (superstructure), and \#60 (substructure), and every calibration step is titled as a different Condition State (CS) to express the bridge component's condition ratings. The CI for an element level inspection method consists of 4 or 5 different CSs (depending on the bridge authorities' adoption and customisation) to quantify condition states of bridge elements.

\subsection{Neural Network Modelling}

Artificial Neural Networks (ANNs) have attracted world-wide attention over the last two decades, and are one of the supreme tools for solving the stated research problem, 
because they are simple and effective for examining data and developing suitable models. It is an emerging technique and a promising tool in process identification and control, owing to its ability to model processes reliability. For the proposed BPM model, ANNs have a great aptitude in terms of determining missing values and patterns in historical datasets. They also have a first-rate ability to derive meaning from complicated or imprecise datasets. They can be used to extract patterns or detect trends in data, which is useful for seizing unknown areas or periods of data patterns. Many different ANN models have been developed to achieve various predictions such as: (1) learning to predict events based on observation of patterns in historical data; (2) learning to classify unseen data into predefined classes based on observations in the characteristics of the data; and (3) learning to cluster the training data into natural groups based on similarity of characteristics [13].

The Back-propagation feed-forward ANN is a universal function approximator that typically yields better results than traditional approximation methods in practical applications. Technically, it normally has two different stages, i.e. the training and testing stages, to induce values as predictions. The training stage is the learning process to detect patterns of interest in the dataset, and then additional patterns, unseen by the network previously, are applied as inputs in the testing stage to produce suitable outputs.

\subsection{Composition of the Proposed ANN model}

Fig.4 illustrates the proposed single-layer feed-forward back-propagation neural network model. The model consists of an input layer, hidden layer(s) and an output layer, whereby neurons exist in the hidden and output layers connected by weights. A neuron in the hidden layer obtains data from the input layer, which is processed by the calculation of a weighted sum and subsequently passed to another neuron in the output layer through a weighted connection.

\section{Fig.4 Structure of ANN-based BPM}

In the proposed model, the number of neurons in the hidden layer can be determined by the number of existing inspection and non-bridge factors in the input and output layer, respectively. The specifications for the inputs, outputs and functions of the proposed BPM are detailed in Table 1. The input layer has 21 variables including 4 factors for the number of vehicles, 2 factors for the population growth and 15 factors for climate. This information is used to train the ANN to determine the correlation with currently available bridge condition rating data in the output layer. The sigmoid function as a typical neuronal non-linear transfer function is used in the proposed model due to its non-linear properties. If a linear transfer function were used in the proposed model, each of the neuronal inputs would become multiplied by their identical proportion during training. It may cause the entire system to generate inappropriate outputs. Hence, the sigmoid transfer function aids to isolate specific input pathways $[14,15]$.

Table 1 The components of the proposed neural network model

As mentioned in Section 1, due to the limitation of bridge element condition rating availability for a BMS's historical requirements, the total eligible data for this study is only a small amount. It is not a sufficient amount for training to construct reliable 
weights using the available dataset, thus additional variables, non-bridge factors, are required, which have a relationship with the training outputs (condition rating data), to support the training output's lack of trend. The selected non-bridge factors are suited for use as variables for bridge condition rating information because bridges are always exposed to the natural environment and traffic conditions. All variables used as nonbridge factors have been available for many years as publicly-accessible data.

\subsection{Sample datasets for BPM}

As mentioned in Section 2.1, non-bridge factors can be used to add trends into existing bridge condition ratings, thus the sensitivity of these factors affects the reliability of backward predictions.

Based on the approximate bridge locations provided from the Maryland DoT, historical vehicle registrations, census population, and climate data have been obtained from the Federal Highway Administration, U.S. Census Bureau, and the U.S. Department of commerce National Oceanic \& Atmospheric Administration, respectively.

The historical vehicle registrations are plotted in Fig.5 (a) in terms of 4 different variables: passenger vehicle, bus, truck and total number of vehicles. The historical population changes are plotted in Fig.5 (b) in terms of 2 different variables: bridge location - city and state. The historical temperature and precipitation changes are plotted in Fig.5 (c) \& (d), respectively.

Fig.5 Raw data of non-bridge factors for BPM inputs

The details of raw datasets for NBI and BMS inputs for the different tests are detailed in Fig.6 and Table 2, respectively. The initial assumption of the BPM is that the components and elements of the bridge have excellent condition ratings when it was built.

Fig.6 Raw data of NBI for BPM outputs (Bridge \#0301xxxx1)

Table 2 Raw data of actual condition ratings (Element \#234 on Bridge \#0301xxxx1)

\subsection{Validation of the BPM}

The entire timeframe of the bridge data used in the BPM is from the year 1966 to 2004. Amongst these, on 5 occasions, inspection results were used as ANN-based BPM training inputs and outputs (from 1996 in 2-year increments to 2004). The assumed condition rating at year zero (1966) of the bridge has also been used. The remaining years (from 1968 to1994 with 2-year increments) of historical condition ratings can be generated by the proposed BPM.

Generated historical condition ratings are compared with existing information to assess the reliability of results. As shown in Fig.7, the timeframe of Tests \#1 and \#2 for the proposed BPM using NBI information is described in Fig. 7 which shows the timeframe of the inputs (Fig.7 (c) and (f)) and their results (Fig.7 (d) and (g)). The 
two different results are compared with the existing NBI (Fig.7 (e) and (h)) to measure the BPM performance using the raw datasets.

Fig.7 BPM timeframe (Test\#1 and 2 using NBI for performance measurements)

NBI data, which are used to generate the historical condition ratings between 1968 and 1994, are employed in the first test. Fig. 8 shows the results of the generated historical condition ratings for decks, superstructures, and substructures. $78.6 \%$ of the generated data can be directly compared with the actual NBI data to measure the prediction errors. There are two different modes (test \#1 and 2) are conducted to validate this model and are detailed in Sections 3.1 and 3.2.

Fig.8 BPM results for Bridge $\# 0312 \times x \times 1$

(Note that the number of prediction results in each year is 66 which is the combined number of learning rates (lr:0.0-0.5) and momentum coefficients (mc:0.0-1.0) in the neural network configuration)

\subsection{Backward Comparisons}

As mentioned, most of the generated data from Test \#1 can be directly compared with the existing historical NBI datasets. The comparisons between the results for each bridge component and its NBI records are plotted in Fig.9. Most artificially-generated historical condition ratings are obtained within a prediction error scale of less than $\pm 10 \%$. However, year 1982 in deck, year 1984 in superstructure, and years 1982, 1984 and 1986 in substructure exhibit larger errors than the maximum allowance $( \pm 10 \%)$. The main reason for generating imprecise outcomes is that as mentioned earlier in Section 2.1, the proposed model was developed based on the correlations of condition ratings and their corresponding non-bridge factors in the ANN training stage. Nevertheless, this is adequate for historical condition ratings, because they are ranked within the same CS $(60 \% \leq \mathrm{CS} 2<80 \%)$.

In the case where the ANN training datasets using existing condition ratings, do not have a relevant correlation with the non-bridge factors, the BPM cannot provide reliable historical condition ratings for a specific year. For example, depreciations of condition ratings caused by sudden physical damages to a bridge are not influenced by the non-bridge factors used in the BPM yielding unreliable predictions.

The results of the backward predictions are validated by comparing them with existing historical condition ratings (Test \#1). However, the actual element-level condition ratings for BMS inputs are only available in a small number of datasets and are not applicable to the backward comparison method used in this section for BPM validation. Therefore, the forward comparison (Test \#2) is used in Section 3.2 to validate the BPM.

Fig.9 Performance measurements: existing NBI vs BPM results (Bridge \# 0312xxx1)

\subsection{Forward Comparisons}

The other validation method for the BPM is conducted in this section, called the forward comparison (Test \#2). The BPM of the ANN training inputs in Test \#2 
utilises the results of Test \#1 between 1968 and 1994, as illustrated in Fig. 7 (f). The BPM produces the future condition ratings between 1996 and 2004. The results are also compared with existing condition ratings (year 1996-2004) and summarised in Table 3. Each year of condition ratings predicted using the ANN-based BPM provides satisfactory results within the error allowances. Therefore, the forward comparison method constructed in this section can be used to validate the BPM results using the actual BMS condition rating inputs.

Table 3 Summary of prediction performance for test 2

\subsection{Minimum Inputs for the BPM}

To verify the minimum BPM input requirements and the response with the number of inspection records in the BPM, additional tests are also conducted for the various years of condition ratings. The BPM assumes that the bridge agency only retained up to 5 sets of historical condition ratings.

Fig.10 shows the forward comparisons when the BPM is used for various years of condition ratings. The figure demonstrates the average prediction errors, which gradually decrease when the number of inspections is increased. The effective range of inputs for the number of inspections is from 2 sets of records, which partially meet the maximum error allowance. It is demonstrated that the proposed BPM can provide satisfactory results when more than 4 sets of inspection records are used as its inputs.

Fig.10 Average errors for different numbers of training inputs (bridge\# 0312xxx1)

\subsection{BPM for BMS Condition Ratings}

The BPM is validated using two different methods as described in Section 3. The $\mathrm{BPM}$ is still required to deal with actual BMS inputs to demonstrate the contribution of the BPM on the research problem.

The BPM test for BMS actual condition rating inputs is conducted by using one element in the superstructure on the same bridge (Bridge\# 3210xxx1) in Section 3. Thus, bridge specifications and its lifecycle are identical. The bridge sample datasets obtained (Element \#234: Reinforced Concrete Pier Cap) are the actual BMS condition rating inputs and have been collected to periodically update the BMS database. In general, the element-level inspection results contain more detailed condition states than the NBI (component level of condition ratings). In the element-level inspection method, the condition ratings of Element \#234 are quantified using five different condition states.

Fig.11 describes the BPM timeframe for the obtained bridge Element \#234. It shows the time in the number of years for: (a) the entire bridge life cycle; (b) available condition ratings; (c) BPM inputs; (d) generated historical condition ratings; (e) inputs for validation; (f) forward-prediction results; and (g) result comparisons of forwardpredictions with the existing condition rating datasets. Only 5 historical condition rating datasets (from 1996 to 2004 with 2-year increments) are available to be used as BPM input values as detailed earlier in Table 2. 
Fig.11 BPM timeframe of Element \#234 in Bridge \#3210xxx1 (Test using BMS inputs for performance measurements)

The average quantity of each CS on this element\#234 between 1996 and 2004 is $80 \%$, $16.2 \%$, and $3.8 \%$ of the total elements in CS1, CS2, and CS3, respectively. The BPM generates historical condition ratings from 1968 to 1994 in three different proportions of the condition state as shown in Fig.12. Fig.12 (c) shows that 3.8\% of total elements have historically deteriorated more than the others. In other words, maintenance activities (repair or replacement) on these numbers of elements have been done historically.

Fig.12 Backward-prediction results for Element \#234 for Bridge \#0312xxx1

(Note that the number of prediction results in each year is 66 which is the combined number of learning rates (lr:0.0-0.5) and momentum coefficients (mc:0.0-1.0) in the neural network configuration)

In addition, the format of the final results has to be modified to conform to the type of element level inspection. The BPM outcomes cannot be used directly as BMS inputs. Hence, the BPM results are required to go through a simple post-calibration process. Table 4 shows an example of BPM results in 1982. The element quantity can be determined by using Equation 1, from that, the yearly element quantity is shown in Table 5. These graph and table represent the BPM results using 5 existing condition rating datasets (1996-2004), which are employed to generate historical condition ratings from 1968 to 1994 with 2-year increments. The average proportion of each CS is also shown.

Table 4 Conversion from BPM results to BMS input format (Year 1982 of Element \#234 for Bridge \#0312xxx1)

Table 5 Results of BPM for BMS inputs

$$
\begin{gathered}
\text { Total quantity } \\
\text { of subjected } \\
\text { element }
\end{gathered}=\left(\begin{array}{ccc}
\text { Proportion of } \\
\text { BPM results } \\
\text { per CS }
\end{array} \quad \begin{array}{c}
\text { Total quantity of } \\
\text { subjected element }
\end{array} \times \begin{array}{c}
\text { Average } \\
\text { proportion of }
\end{array}\right)
$$

\subsection{Performance Measurements}

To validate the BPM outcomes (1968-1994), the forward comparison method (Test\#2) is used. This validation method already tested in earlier BPM modelling has been described by using NBI datasets in Section 3.2. This is because condition ratings between 1967 and 1994 are not available for direct comparison.

For the forward comparison (Test \#2), the back-prediction results (1968-1994) are used as input datasets in this test to generate the condition ratings for the present years (1996-2004). The BPM generated condition ratings are then directly compared with existing condition rating datasets. The BPM results are shown in Fig. 13 in four different proportions of element quantity.

Fig.13 Performance measurements of Element \#234 for Bridge \#0312xxx1 
(Note that the number of prediction results in each year is 66 which is the combined number of learning rates (1r:0.0-0.5) and momentum coefficients (mc:0.0-1.0) in the neural network configuration)

The identical calibration processes using Equation 1 are performed to conform to the type of element-level inspection format as shown in Table 4. Table 6 shows the final results from the BPM as well as its prediction errors. The yearly average prediction errors are less than $\pm 10 \%$, which is acceptable. Therefore, the generated historical condition ratings (1966-1994) by the BPM can be used as historical condition ratings.

Table 6 Prediction errors of the BPM using forward comparisons

\subsection{Case Study}

This section provides brief descriptions and results of the case study. This case study was conducted to present an additional validation for the BPM.

The obtained bridge sample datasets and its corresponding years of non-bridge factors including climates and population growth for these case studies are provided by Road and Traffic Authority New South Wales (RTA NSW), the Australian Government Bureau of Meteorology and the Australian Bureau of Statistics (ABS).

RTA NSW is one of the earlier BMS adopters among bridge authorities in Australia and has utilised the PONTIS BMS software, which is based on an element-level inspection method to collect bridge condition ratings. The main reason for adopting their bridge sample data was that they hold the largest quantity of bridge element condition rating datasets for BMS software in Australia.

RTA NSW provided 10 different bridge sample data with 15 different types of bridge elements. Most given bridges were built during the 1960s and 1970s, with an approximate average life cycle of 40 years.

The number of bridges element inspection datasets obtained and used for the BPM is mostly between 4 to 6 of the inspection records for the 10 to 12 years of historical bridge condition ratings. The most typical bridge element types selected for the case studies are defined in the RTA bridge inspection procedure manual [16] as detailed in Table 7. The total number of the most typical bridge elements used in the case studies is nine from seven bridges.

\section{Table $7 \quad$ Typical bridge elements tested}

The selected bridge elements have 3 to 5 different condition states (CSs) for the evaluation of their bridge element condition ratings. As illustrated in Fig.14, all tested actual condition states are calibrated to suit the BPM input environment.

Fig.14 Scales of bridge condition states (CSs) for the BPM

Similar types of non-bridge factors (as outlined in Section $3 \& 4$ ) are employed for the BPM's training data, such as historical population growth and climates surrounding the bridge area. Two historical population variables (city and state population growth) and 5 different climate variables (maximum temperatures $>40{ }^{\circ} \mathrm{C}$, maximum temperatures $>35^{\circ} \mathrm{C}$, minimum temperature $<0{ }^{\circ} \mathrm{C}$, highest maximum 
temperature, lowest minimum temperature) were used as non-bridge factors in this case study.

Among the selected bridge elements in the case study, this section demonstrates one of 3-CS's bridge elements (the element code BELA: Elastomeric Bearing Pad). The available input datasets for the BPM consists of 5 inspections as detailed in Table 8 and have 1-2 year regular inspection intervals. The timeframe for the case study of element code BELA is described in Fig. 15 showing the time in number of years for: (a) the entire bridge life cycle; (b) available condition ratings; (c) BPM inputs; (d) generated historical condition ratings; (e) inputs for validation; (f) forward-prediction results; and (g) result comparisons of forward-predictions with the existing condition rating datasets.

Table 8 Element condition ratings (3-CSs) obtained for the case study

Fig.15 BPM timeframe for Element code BELA (Bridge\# 5xx0)

\subsection{BPM Modelling and Results}

The average quantity of each CS on this element between 1994-2005 is concentrated on CS1 showing $100 \%$ of the total elements. The BPM generates historical condition ratings from 1974 to 1992, with 2-year increments using 100\% proportions of CS1.

The BPM generated 10 historical condition ratings for the past 20 years from 1974 to 1992 of Element code BELA. The yearly average predictions are shown in Fig.16. Most past conditions are positioned in CS1 and CS2. However, there are some major condition improvements between 1980-1982 and 1988-1991, which are observed from the backward prediction results.

\section{Fig.16 Backward prediction results for Element BELA on Bridge\# 5xx0}

The outcomes from neural network modelling cannot be used directly as BMS database inputs. The format of BPM results has to be calibrated to conform to the element level inspection format. Thus, generated past condition ratings require postcalibrations using Equation 1 as given in Section 4.0. As a result, the yearly numbers of elements from 1974 to 1992 per condition state can be determined. These results are presented in Table 9 in accordance with the BMS software input format.

Table 9 BPM results as BMS inputs (Element BELA for Bridge \#5xx0)

\subsection{Evaluation of BPM prediction}

Another neural network model is required for BPM verification. The validation of all elements obtained is conducted using the second validation method (generated historical condition rating datasets are used as input datasets for this neural network training) presented in Section 4. This is because this particular type of inspection method was not previously employed, hence, direct comparisons between generated and existing datasets are not possible. However, as shown in Section 4, the forward comparison method is validated to measure the BPM prediction accuracy.

The ANN training datasets used the BPM results collected from 1973 to 1992 including the assumed element condition ratings at the year of bridge construction as 
shown in Section 5.1. To compare these condition ratings with the existing datasets, results from the neural network model are converted to follow the format of the element-level inspection method using Equation 1.

As a result, in CS1, accurate results using the neural network model for this element are obtained as shown in Fig.17 (a). Similarly, Fig.17 (b) and (c) shows respectively the acceptable prediction errors in CS2 and CS3 of up to about $2.5 \%$. The maximum error in 3-CS's element condition ratings are about 33\% $( \pm 16.5 \%)$.

Fig.17 Comparisons on each condition state between forward-manner prediction results and existing condition ratings from 1994 to 2005 for Bridge \#5xx0

However, using the prediction errors in CS1, the actual number of elements is greater than the predicted number of elements as shown in Table 10. In other words, the prediction results present a lower risk. Consequently, the generated historical element condition ratings for Element code BELA are acceptable to be recorded in the BMS database as historical bridge inspection records.

Table 10 Comparisons between forward-predictions and existing condition ratings for Bridge $\# 5 \times x 0$

\subsection{Summary of case studies}

The other typical bridge elements are modelled to demonstrate the capability of the BPM. The test methods, which generate the missing bridge elements' condition ratings and their validations, are identical to those presented in Section 5. As shown in Table 11, the overall results from the case studies meet the pre-defined maximum prediction errors for the nine bridge elements. The maximum average yearly prediction errors are found to be $10.21 \%, 9.26 \%$ and $4.40 \%$ respectively for the three different condition states (CSs). These BPM results are considered satisfactory.

Table 11 Average condition rating differences of the 9 typical bridge elements

\subsection{Discussion}

The development of the ANN-based Backward Prediction Model (BPM) is described in this paper. The BPM uses non-bridge factors as supplementary historical data to overcome the lack of historical bridge data in terms of its quantity and patterns. The non-bridge factors including local climates, traffic volume and population growth in the area surrounding the bridge are employed to help establish the correlations between the non-bridge factors and the lack of historical data patterns in the existing condition ratings.

To establish the methodology of backward manner prediction, sample structural condition rating datasets made available by the Maryland Department of Transportation (Maryland DoT), and based on five existing condition rating datasets (or $26 \%$ of the total record), ensured that the BPM was able to generate 14 missing datasets (or $74 \%$ ) for the intervening years when proper inspection records were missing. The average ratio of the generated and existing datasets is about 3 . The average prediction errors of the generated bridge condition ratings are between $6.7 \%$ $7.5 \%$ over a period of 20 years. 
To confirm the BPM methodology established, case studies were performed using 9 typical bridge elements from the Road and Traffic Authority of New South Wales (RTA NSW). Test methods to generate missing bridge elements' condition ratings and their validation methods were identical to those presented in the pilot study. The maximum yearly prediction errors of three different condition state scales (CS 3, 4 and 5) are $18.61 \%, 23.89 \%$ and $4.40 \%$ respectively. These are satisfactory as compared to the maximum errors of $33.33 \%, 25 \%$ and $20 \%$ for $3 \mathrm{CSs}, 4 \mathrm{CSs}$ and $5 \mathrm{CSs}$, respectively.

In addition, the methodology described in this paper aims to establish a possible solution and a very initial approach for determining the unavailable historical structural condition ratings. Further study may be required to explore the types and numbers of non-bridge factors for the BPM. As further critical non-bridge factors are identified and incorporated into the model, the accuracy of correlations with a small number of structural condition ratings, as well as future condition rating predictions, will improve.

\subsection{Conclusions}

It is generally recognised that for most bridges there are big time gaps between the dates of construction, adoption and implementation of relevant Bridge Management Systems (BMSs). As a result, suitable bridge inspection records, and of course the bridge element condition rating datasets, are missing or unavailable for the intervening years. The missing data, which is required as input to the relevant analysis modules of any BMS, is a major factor contributing to the unreliable BMS outcomes currently experienced by most bridge agencies.

The main focus of the proposed research is to rectify this untenable situation faced by these agencies in most countries. To this end, the so-called Back Prediction Model (BPM) is developed as part of this research effort. Capable of generating the missing data, the BPM incorporates ANN techniques and operates on the limited existing inspection records and historical non-bridge factors such as local climates, number of vehicles and population growth in the area surrounding the bridge. A total of nine case studies conducted in this research confirms the reliability of the BPM to help produce accurate BMS outcomes.

In conclusion, the main goal of this research is to develop an appropriate methodology for an effective bridge condition rating model to produce the historical data of the relevant bridge elements. The BPM is believed to be a useful methodology for an effective implementation of BMS packages and deserves wider application. However further insight into the appropriate non-bridge factors is required as recommended below:

- Parametric studies should be conducted to determine the optimum numbers and types of non-bridge factors for different locations and types of bridges. This should lead to more effective operations of the BPM by excluding the unnecessary nonbridge factors to yield a superior correlation between condition ratings and the wellchosen non-bridge factors. 
- Development of a Long-term Bridge Performance (LTBP) model should be carried out using a comprehensive structural condition rating database. This should lead to more reliable future structural condition rating predictions. This will also enhance the reliable outcomes of many other analytical BMS modules.

\section{Acknowledgements}

The resources used for the present study were provided by Maryland State Department of Transportation, U.S. The writers wish to thank Mr. Earle Freedman and Matt Zulkowski from Maryland Department of Transportation for tracking our information requests. Authors also would like to thank Mr. Perumynar Siva of the New South Wales Road and Traffic Authority, who has provided the important historical bridge datasets which enabled the case studies to be successfully completed. In particular, Professor Waheed Uddin from The University of Mississippi for insightful comments and suggestions.

$\begin{array}{ll}\text { Abbreviations } \\ \text { ANN } & \text { Artificial Neural Network } \\ \text { BMS } & \text { Bridge Management System } \\ \text { BPM } & \text { Backward Prediction Model } \\ \text { CI } & \text { Condition Index } \\ \text { CS } & \text { Condition State } \\ \text { DB } & \text { Database } \\ \text { DoT } & \text { Department of Transportation } \\ \text { IMS } & \text { Infrastructure Management System } \\ \text { MR\&R } & \text { Maintenance, Repair \& Rehabilitation } \\ \text { NBI } & \text { National Bridge Inventory }\end{array}$

\section{References}

[1] D.M. Frangopol, E.S. Gharaibeh, J.S. Kong and M. Miyake, Optimal NetworkLevel Bridge Maintenance Planning Based on Minimum Expected Cost, In the Proce. of the Transportation Research Record, Florida, (2000) 26-33.

[2] S. Madanat, Optimal infrastructure management decisions under uncertainty, Transportation Research Part. C., 1 (1993) 77-88.

[3] G. Hearn, Segmental Inspection for Improved Condition Reporting in BMS, In the Proce. of the Eighth Int. Bridge Management Conference, Denver, Colorado, (1999) B-3/1-8.

[4] B. Godart and P.R. Vassie, Review of existing BMS and definition of inputs for the proposed BMS, Deliverable D4: BRIME Report (1999 a).

[5] G. Hearn, R.L. Purvis, P.D. Thompson, W.H. Bushman, K.K. McGhee and W.T. McKeel, Bridge Maintenance and Management: A look to the future, In the Proce. of the TRB 81st Annual Meeting: A3C06:Structures Maintenance and Management (2000) 1-7.

[6] A. Weigend and N. Gershenfeld, Time Series Prediction: Forecasting the Futureand Understanding the Past, Addison-Wesley, Reading, MA, (1994) 5966.

[7] B.G. Tabachinick and L.S. Fidell, Using Multivariate Statistics, Allyn and Bacon (2001). 
[8] T. Karna, F. Rossi and A. Lendasse, LS-SVM functional network for time series prediction, In the Proce. of the of XIV ${ }^{\text {th }}$ European Symposium on Artificial Neural Networks (ESANN 2006), Bruges. Belgium, (2006) 473-478.

[9] R.G. Mishalani and M.R. McCord, Infrastructure Condition Assessment, Deterioration Modeling and Maintenance Decision Making: Methodological Advances and Practical Considerations, Journal of Infrastructure Systems, 12(3) (2006) 145-146.

[10] E.P. Small, T. Philbin, M. Fraher and G.P. Romack, Current Status of Bridge Management System Implementation in the United States, Eighth International Bridge Management Conference, Denver, Colorado, (1999) A-1/1-16.

[11] J.H. Milligan, R.J. Nielsen and E.R. Schmeckpeper, Implementing PONTIS As a Bridge Management Tool in Idaho, N04-04, National Institute for Advanced Transportation Technology, University of Idaho, (2004) 2-5.

[12] Federal Highway Administration, Element Level Bridge Inspection (Bridge Management and Inspection Technologies), FHWA-RC-BAL-04-0015, Washington DC (2006).

[13] A.K. Smith, Introduction to neural networks and data mining for business applications, Melbourne: Emerald, Vic. Eruditions Publishing (1999).

[14] J. Anderson, An Introduction to Neural Networks, The MIT Press, Cambridge, MA. (1995).

[15] M. Nelson and W. Illinworth, A Practical Guide to Neural Nets, AddisonWesley Publishing Company, Reading, MA, (1990) 104.

[16] Road and Traffic Authority of New South Wales, RTA Bridge Inspection Procedure, RTA NSW (1999).

\section{Legend of Figures}

Fig.1 Re-illustration of relationships between historical bridge inspection data and BMS outputs [4]

(Note that the relationships with other input sources and BMS outputs are omitted)

Fig.2 Outline of proposed Backward Prediction Model (BPM)

Fig.3 Scale of the condition ratings used in the BPM model

Fig.3 (a) NBI

Fig.3 (b) BMS element condition ratings

Fig.4 Structure of ANN-based BPM

Fig.5 Raw data of non-bridge factors for BPM inputs

Fig.5 (a) Historical vehicle change

Fig.5 (b) Historical population change

Fig.5 (c) Historical Temperature change 
(MMXT: Mean Maximum; MMNT: Mean Minimum; MNTM:

Mean; EMXT: Highest; EMNP: Lowest; DT90: Max. Number of day $\geq 90^{\circ} \mathrm{F}$; DX32: Max. Number of day $\leq 32^{\circ} \mathrm{F}$; DT32: Min. Number of day $\leq 32^{\circ} \mathrm{F}$; DT00: Min. Number of day $\leq 0^{\circ} \mathrm{F}$ )

Fig.5 (d) Historical precipitation change

(TPCP: Total Precipitation; TSNW: Snow and Sleet Total Fall;

MXSD: Snow and Sleet Max. Depth; DP01: Number of Days $\geq 0.1$; DP05: Number of Days $\geq 0.5$; DP10: Number of Days $\geq 1.0$ )

Fig.6 Raw data of NBI for BPM outputs (Bridge \#0301 xxxx1)

Fig.7 BPM timeframe (Test\#1 and 2 using NBI for performance measurements)

Fig.8 BPM results for Bridge $\# 0312 \times x x 1$

Fig. 8 (a) Deck

Fig.8 (b) Superstructure

Fig.8 (c) Substructure

Fig.9 Performance measurements: existing NBI vs BPM results (Bridge \# $0312 \times x \times 1)$

Fig.9 (a) Deck

Fig.9 (b) Superstructure

Fig.9 (c) Substructure

Fig.10 Average errors for different numbers of training inputs (bridge\# $0312 \times x \times 1$ )

Fig.11 BPM timeframe of Element \#234 in Bridge \#3210xxx1 (Test using BMS inputs for performance measurements)

Fig.12 Backward-prediction results for Element \#234 for Bridge \#0312xxx1

(Note that the number of prediction results in each year is 66 which is the combined number of learning rates (1r:0.0-0.5) and momentum coefficients (mc:0.0-1.0) in the neural network configuration)

Fig. 12 (a) About $80 \%$ of the total quantity

Fig. 12 (b) About $16.2 \%$ of the total quantity

Fig. 12 (c) About $3.8 \%$ of the total quantity

Fig.13 Performance measurements of Element \#234 for Bridge \#0312xxx1

(Note that the number of prediction results in each year is 66 which is the combined number of learning rates (1r:0.0-0.5) and momentum coefficients (mc:0.0-1.0) in the neural network configuration)

Fig. 13 (a) About $84.20 \%$ of the total quantity

Fig. 13 (b) About $14.16 \%$ of the total quantity

Fig. 13 (c) About $1.62 \%$ of the total quantity

Fig. 13 (d) About $0.02 \%$ of the total quantity

Fig.14 Scales of bridge condition states (CSs) for the BPM

Fig.15 BPM timeframe for Element code BELA (Bridge\# 5xx0) 
Fig.16 Backward prediction results for Element BELA on Bridge\# 5xx0

(Note that the number of prediction results in each year is 66 which is the combined number of learning rates (1r:0.0-0.5) and momentum coefficients (mc:0.0-1.0) in the neural network configuration)

Fig.17 Comparisons on each condition state between forward-manner prediction results and existing condition ratings from 1994 to 2005 for Bridge \#5xx0 Fig. 17 (a) CS1

Fig. 17 (b) CS2

Fig. 17 (c) CS3

\section{Legend of Tables}

Table 1 The components of the proposed neural network model

Table 2 Raw data of actual condition ratings (Element \#234 on Bridge \#0301xxxx1)

Table 3 Summary of prediction performance for test 2

Table 4 Conversion from BPM results to BMS input format (Year 1982 of Element \#234 for Bridge \#0312xxx1)

Table 5 Results of BPM for BMS inputs

Table 6 Prediction errors of the BPM using forward comparisons

Table $7 \quad$ Typical bridge elements tested

Table 8 Element condition ratings (3CSs) obtained for the case study 
Table 9 BPM results as BMS inputs (Element BELA for Bridge \#5xx0)

Table 10 Comparisons between forward-predictions and existing condition ratings for Bridge $\# 5 \times x 0$

Table 11 Average condition rating differences of the 9 typical bridge elements 


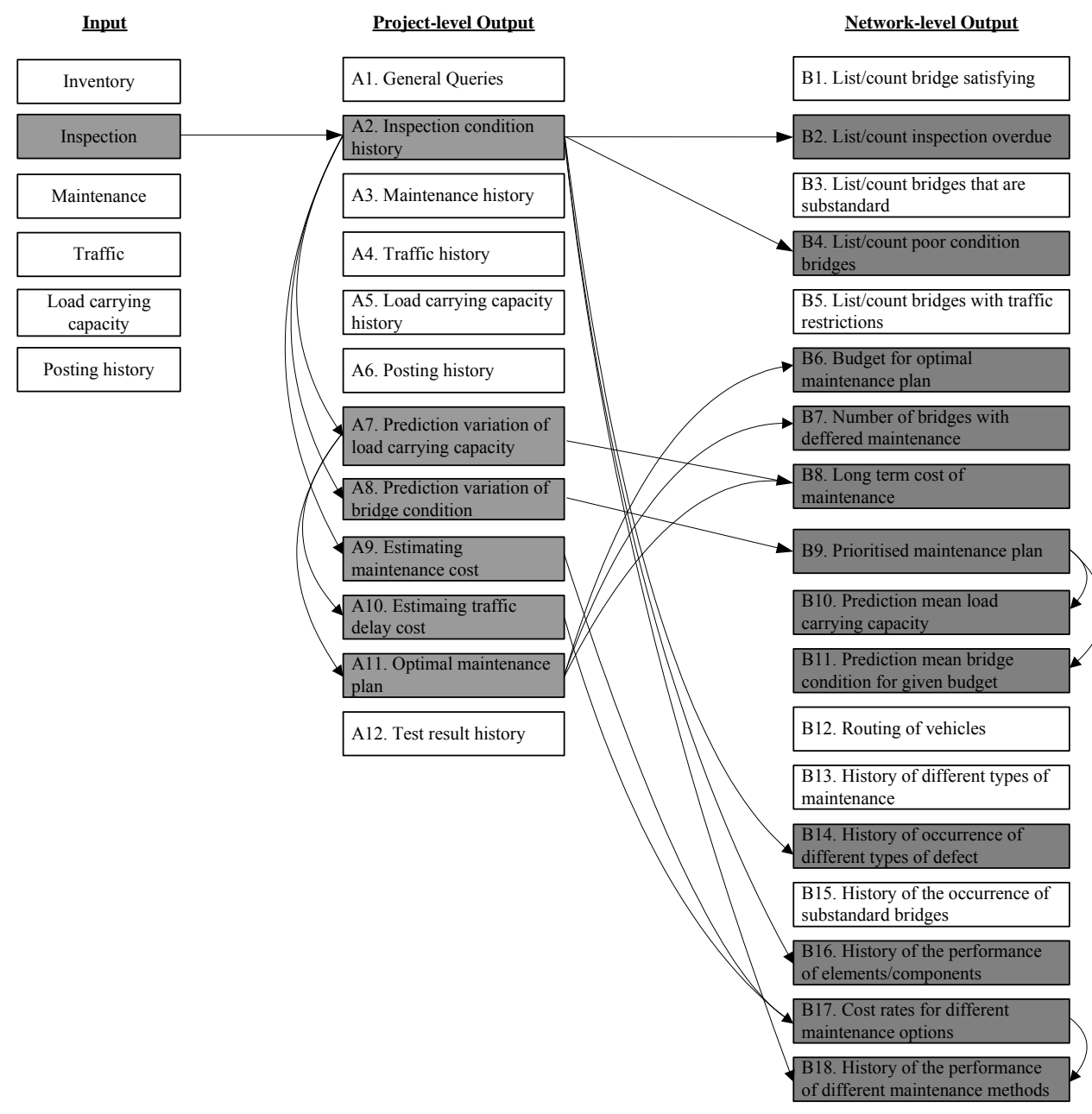

Fig.1. Re-illustration of relationships between historical bridge inspection data and BMS outputs (Godart and Vassie, 1999 a)

(Note that the relationships with other input sources and BMS outputs are omitted) 


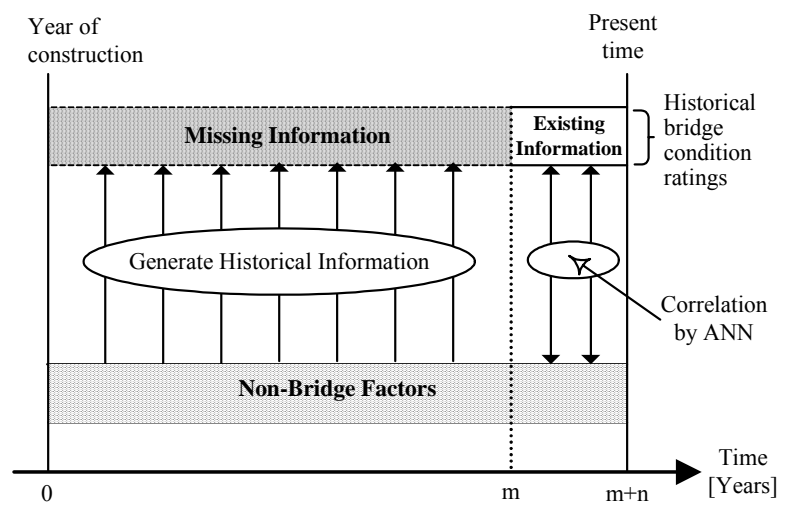

Fig.2. Outline of proposed Backward Prediction Model (BPM) 


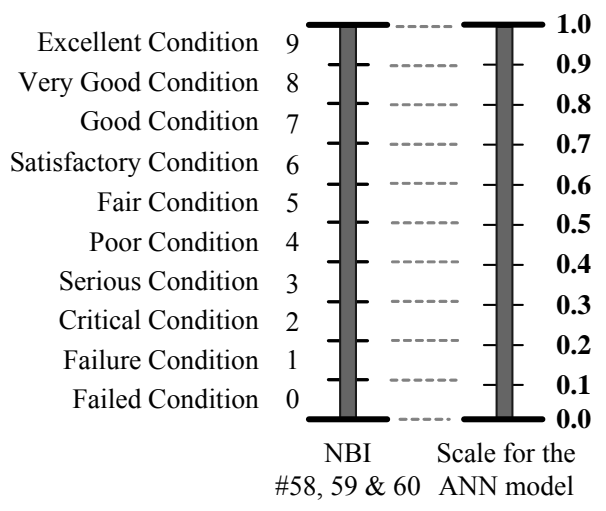

(a) NBI

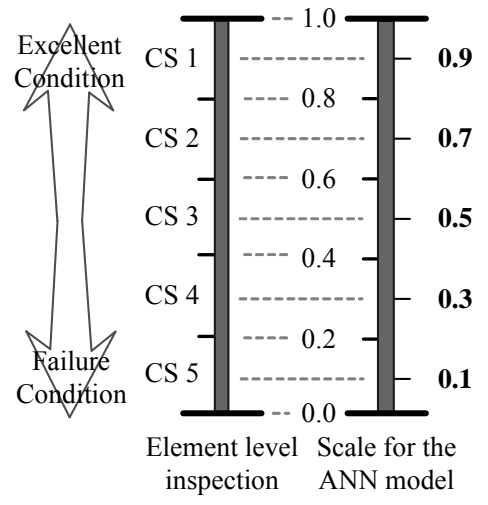

(b) BMS element condition ratings

Fig.3. Scale of the condition ratings used in the BPM model 


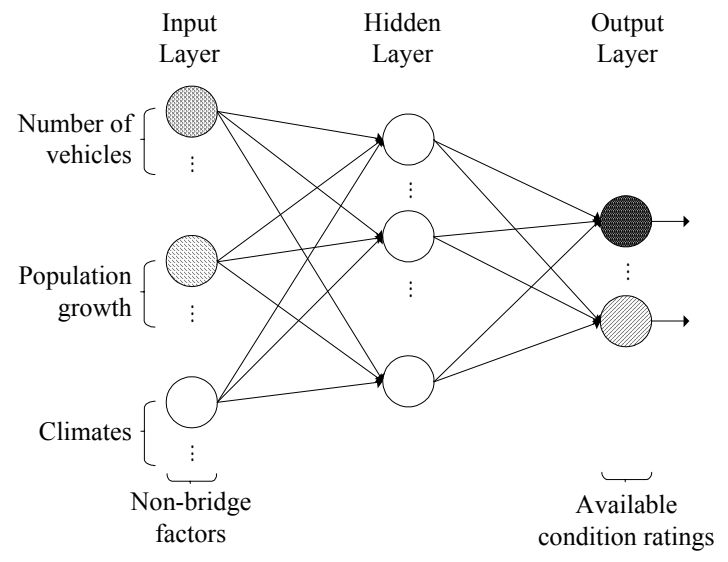

Fig.4. Structure of ANN-based BPM 


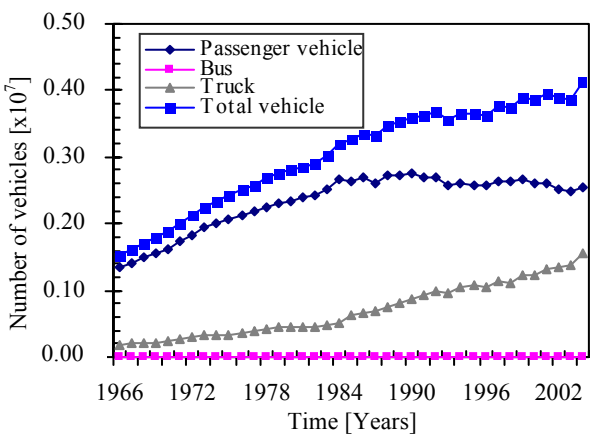

(a) Historical vehicle change

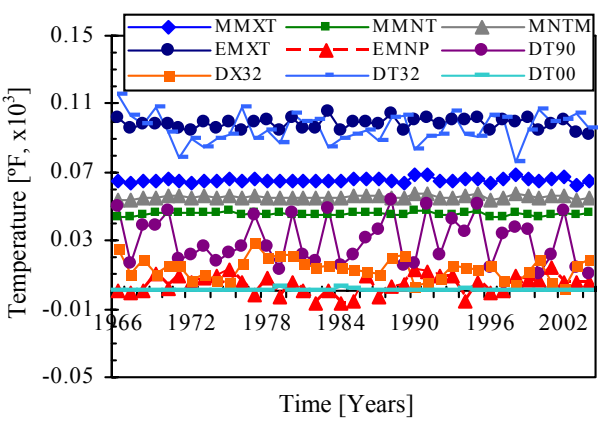

(c) Historical climate change (MMXT: Mean Maximum; MMNT: Mean Minimum; MNTM: Mean; EMXT: Highest; EMNP: Lowest; DT90: Max. Number of day $\geq 90^{\circ} \mathrm{F}$; DX32: Max. Number of day $\leq 32^{\circ} \mathrm{F}$; DT32: Min. Number of day $\leq 32^{\circ} \mathrm{F}$; DT00: Min. Number of day $\leq 0^{\circ} \mathrm{F}$ )

Fig.5. Raw data of non-bridge factors for BPM inputs

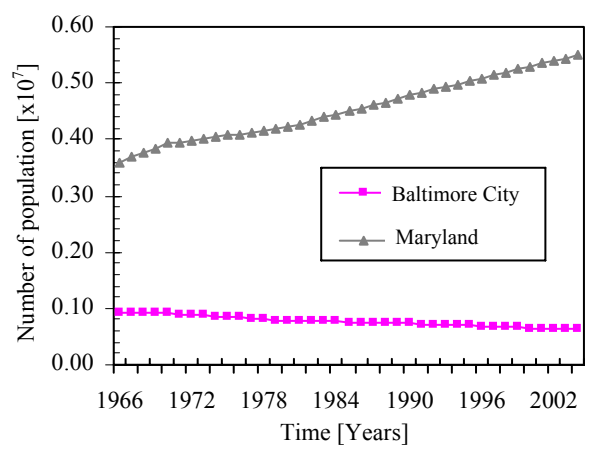

(b) Historical population change

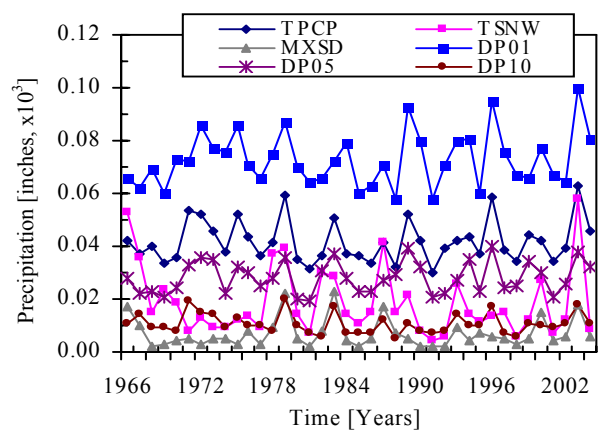

(d) Historical precipitation change (TPCP: Total Precipitation; TSNW: Snow and Sleet Total Fall; MXSD: Snow and Sleet Max. Depth; DP01: Number of Days $\geq 0.1$; DP05: Number of Days $\geq 0.5$; DP10: Number of Days $\geq$ 1.0) 


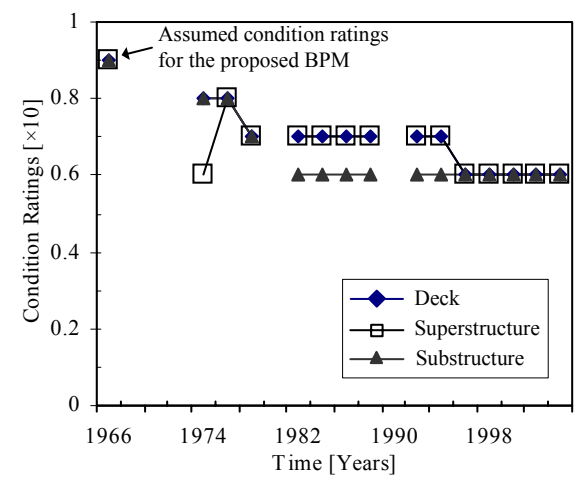

Fig.6. Raw data of NBI for BPM outputs (Bridge \#0301xxxx1) 


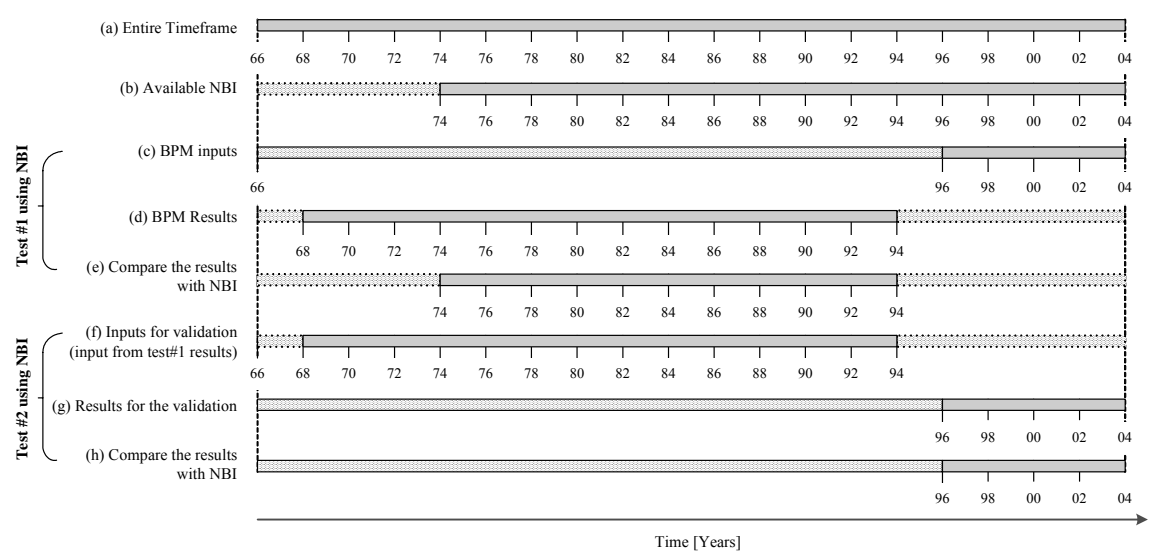

Fig.7. BPM timeframe (Test\#1 and 2 using NBI for performance measurements) 


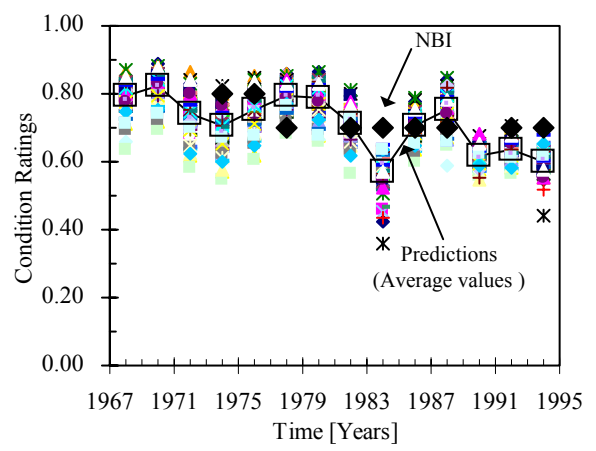

(a) Deck

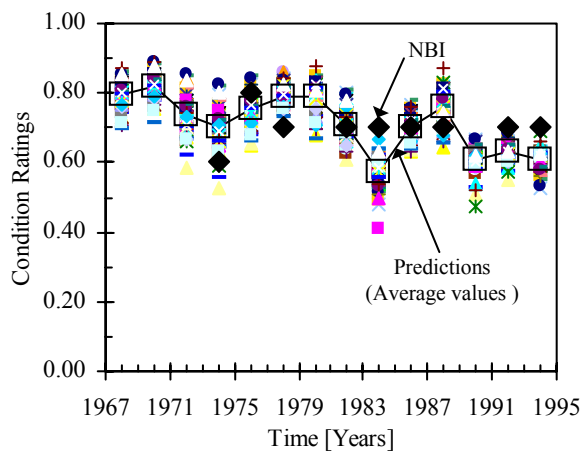

(b) Superstructure

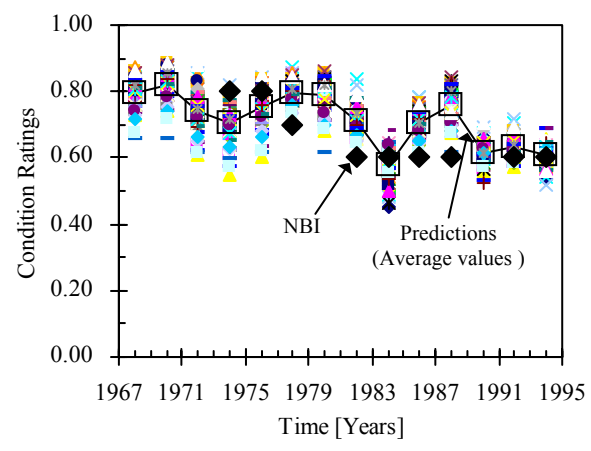

(c) Substructure

Fig.8. BPM results for Bridge $\# 0312 \times x x 1$

(Note that the number of prediction results in each year is 66 which is the combined number of learning rates (lr:0.0-0.5) and momentum coefficients (mc:0.0-1.0) in the neural network configuration) 


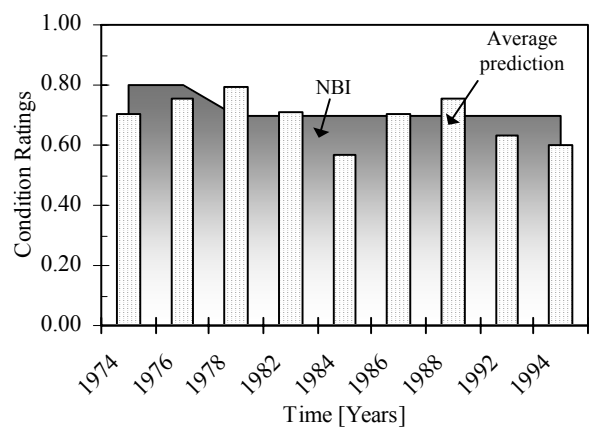

(a) Deck

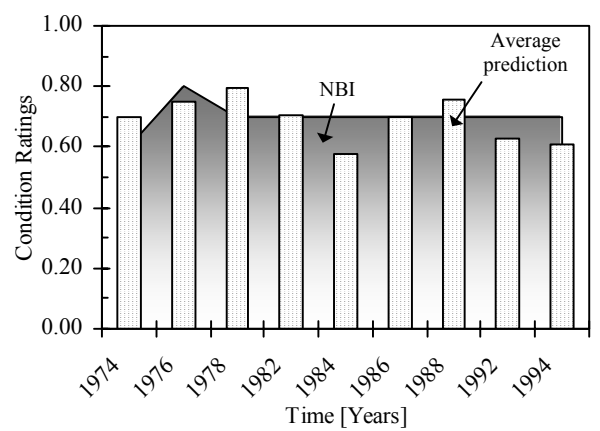

(b) Superstructure

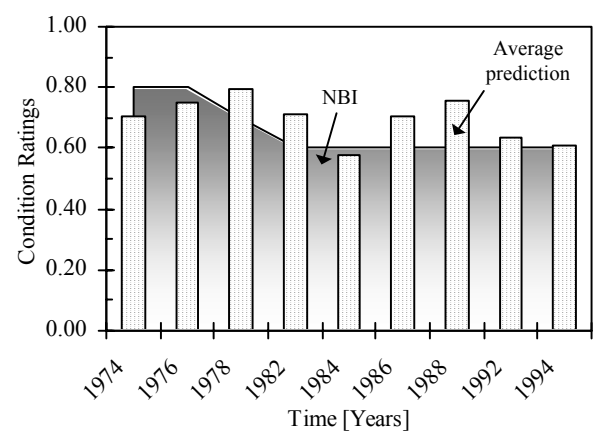

(c) Substructure

Fig.9. Performance measurements: existing NBI vs BPM results (Bridge \# $0312 \times x \times 1)$ 


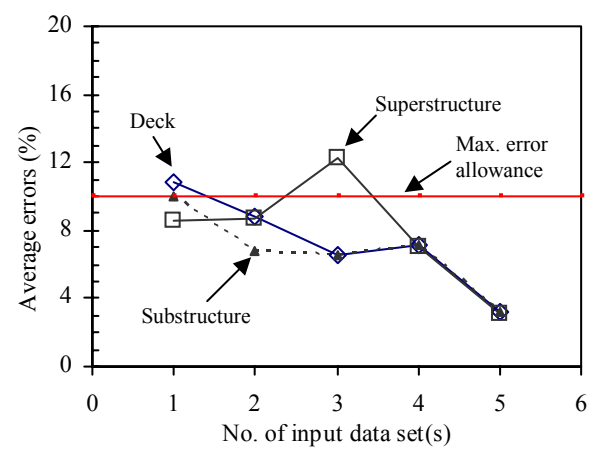

Fig.10 Average errors for different numbers of training inputs (bridge\# 0312xxx1) 


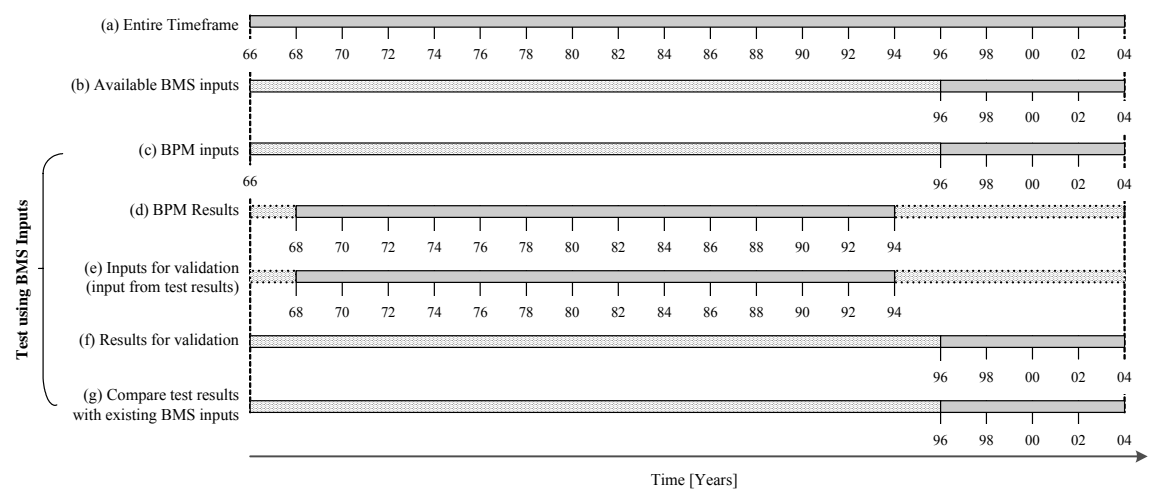

Fig.11. BPM timeframe of Element \#234 in Bridge \#3210xxx1 (Test using BMS inputs for performance measurements) 


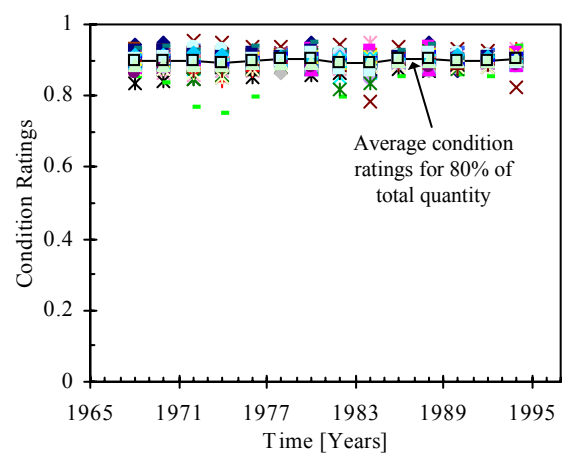

(a) About $80 \%$ of the total quantity

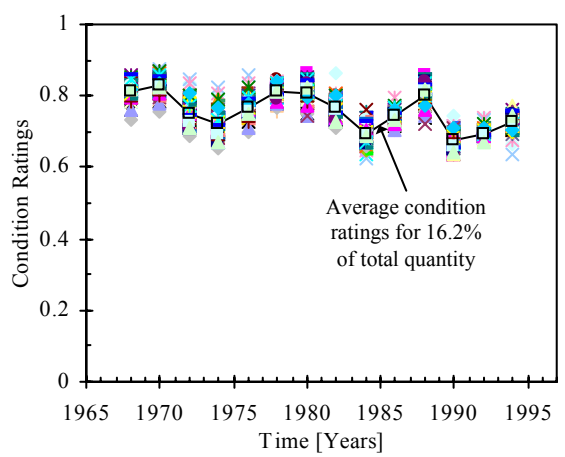

(b) About $16.2 \%$ of the total quantity

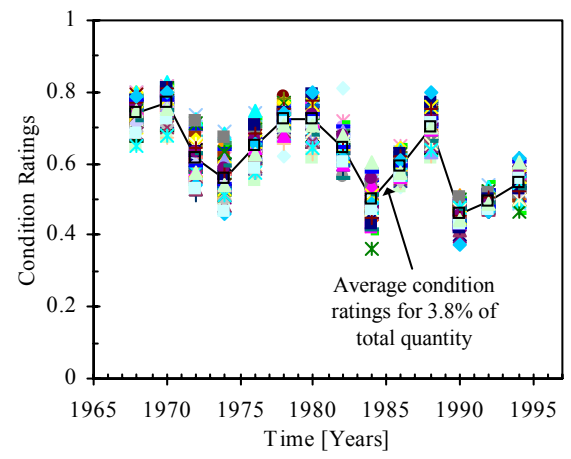

(c) About $3.8 \%$ of the total quantity

Fig. 12. Backward-prediction results for Element \#234 for Bridge \#0312xxx1

(Note that the number of prediction results in each year is 66 which is the combined number of learning rates (lr:0.0-0.5) and momentum coefficients (mc:0.0-1.0) in the neural network configuration) 


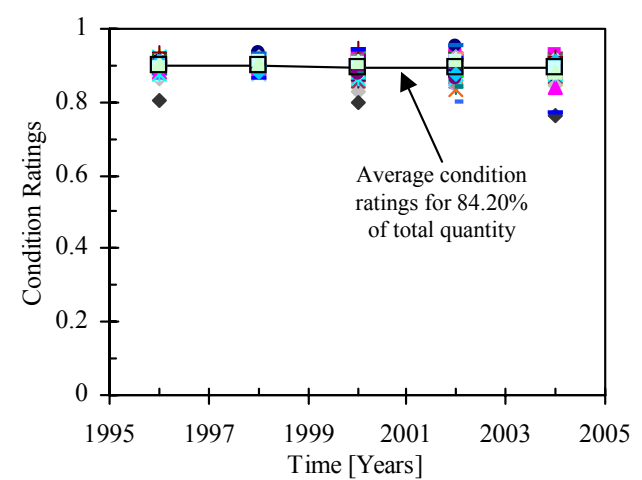

(a) About $84.20 \%$ of the total quantity

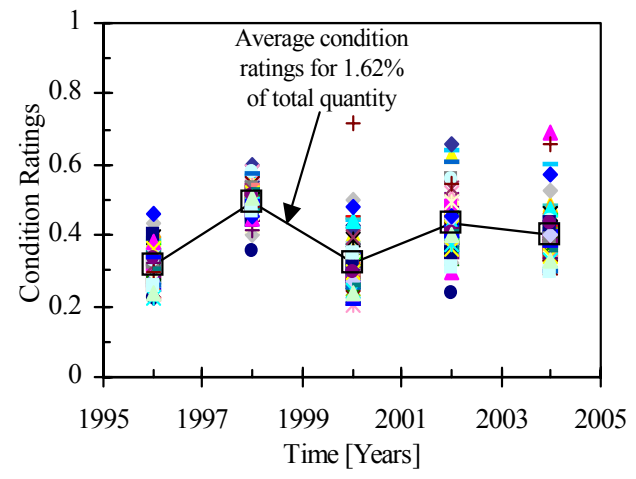

(c) About $1.62 \%$ of the total quantity

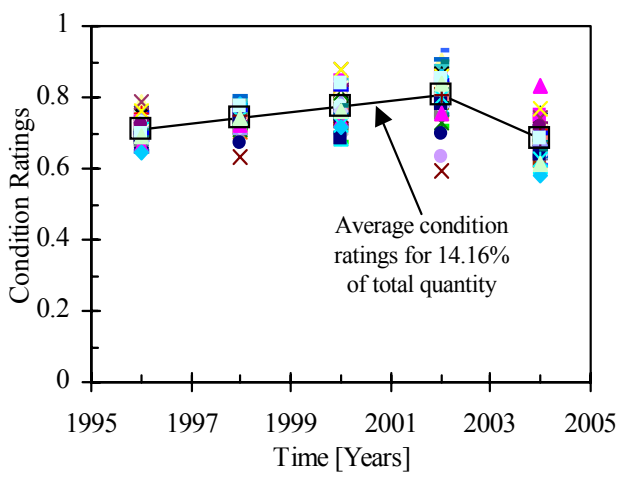

(b) About $14.16 \%$ of the total quantity

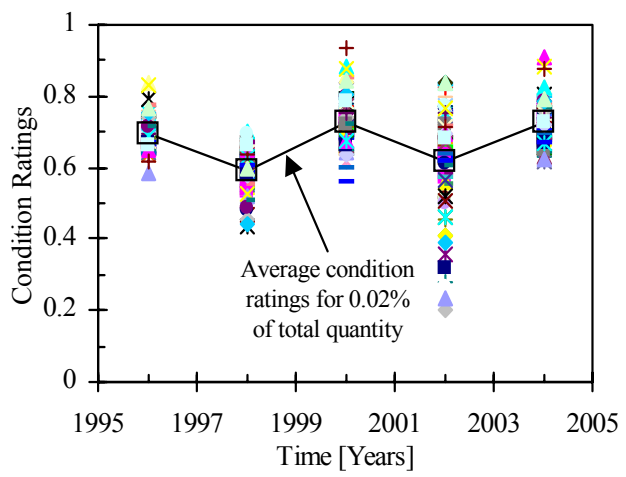

(d) About $0.02 \%$ of the total quantity

Fig. 13. Performance measurements of Element \#234 for Bridge \#0312xxx1 (Note that the number of prediction results in each year is 66 which is the combined number of learning rates (lr:0.0-0.5) and momentum coefficients (mc:0.0-1.0) in the neural network configuration) 

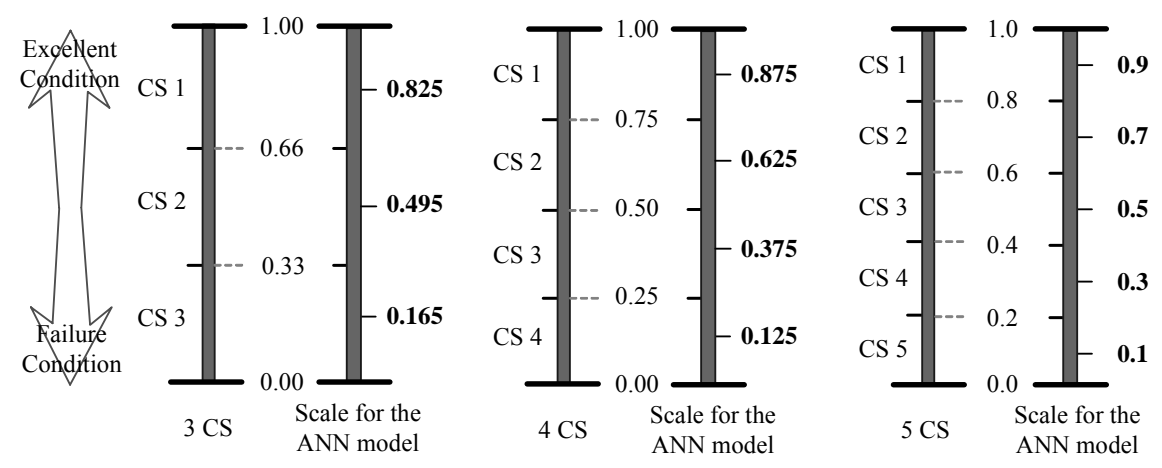

Fig.14 Scales of bridge condition states (CSs) for the BPM 


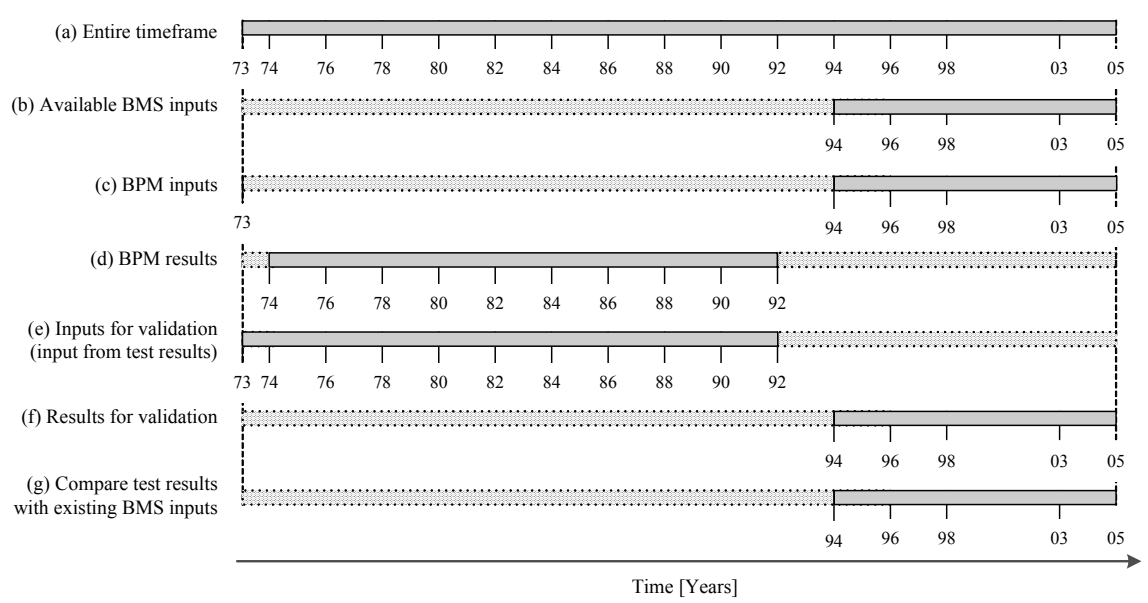

Fig.15 BPM timeframe for Element code BELA (Bridge\# 5xx0) 


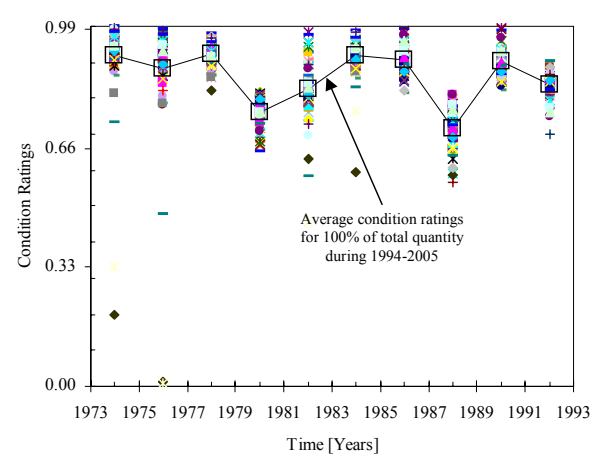

Fig.16 Backward prediction results for Element BELA on Bridge\# 5xx0

(Note that the number of prediction results in each year is 66 which is the combined number of learning rates (lr:0.0-0.5) and momentum coefficients (mc:0.0-1.0) in the neural network configuration) 


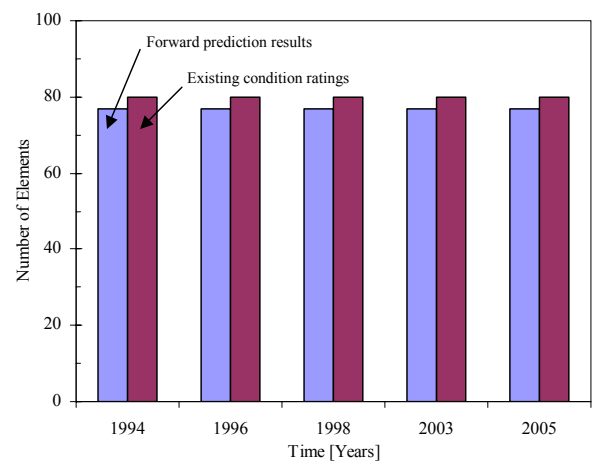

(a) CS1

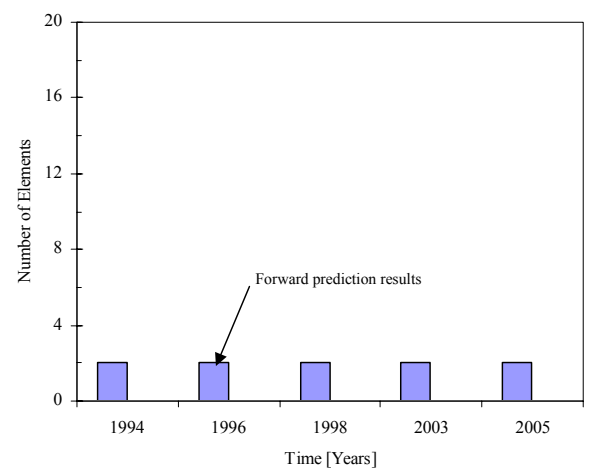

(b) CS2

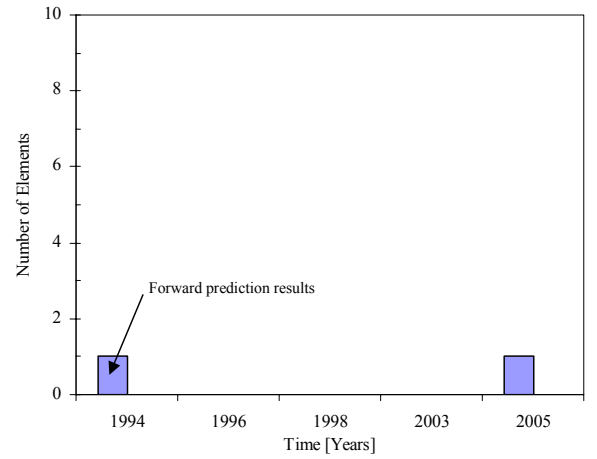

(c) CS3

Fig.17 Comparisons on each condition state between forward-manner prediction results and existing condition ratings from 1994 to 2005 for Bridge \#5xx0 
Table 1. The components of the proposed neural network model

\begin{tabular}{ll}
\hline \hline Training Algorithm & Back-propagation feed-forward \\
Transfer Function & Log-sigmoid function \\
Inputs & Number of vehicles (4 factors) \\
& Population growth (2 factors) \\
& Climates (15 factors) \\
& Bridge condition ratings (1 output) \\
\hline
\end{tabular}


Table 2. Raw data of actual condition ratings (Element \#234 on Bridge \#0301xxxx1)

\begin{tabular}{ccccccc}
\hline \hline $\begin{array}{c}\text { Year of } \\
\text { inspection }\end{array}$ & $\begin{array}{c}\text { Total } \\
\text { quantity } \\
(\%)\end{array}$ & $\begin{array}{c}\text { CS1 } \\
(\%)\end{array}$ & $\begin{array}{c}\text { CS2 } \\
(\%)\end{array}$ & $\begin{array}{c}\text { CS3 } \\
(\%)\end{array}$ & $\begin{array}{c}\text { CS4 } \\
(\%)\end{array}$ & $\begin{array}{c}\text { CS5 } \\
(\%)\end{array}$ \\
\hline \multirow{2}{*}{1996} & 350 & 280 & 50 & 20 & 0 & 0 \\
& $(100)$ & $(80)$ & $(14)$ & $(6)$ & & \\
1998 & 350 & 280 & 50 & 20 & 0 & 0 \\
& $(100)$ & $(80)$ & $(14)$ & $(6)$ & & \\
2000 & 350 & 280 & 50 & 20 & 0 & 0 \\
2002 & $(100)$ & $(80)$ & $(14)$ & $(6)$ & & \\
& 350 & 283 & 67 & 3 & 0 & 0 \\
2004 & $(100)$ & $(80)$ & $(19)$ & $(1)$ & & \\
& 350 & 283 & 67 & 3 & 0 & 0 \\
\hline
\end{tabular}


Table 3. Summary of prediction performance for test 2

\begin{tabular}{clrrr}
\hline \hline Year & Bridge & $\begin{array}{r}\text { NBI } \\
\text { component }\end{array}$ & $\begin{array}{r}\text { Average } \\
\text { rediction }\end{array}$ & Difference \\
\hline \multirow{2}{*}{1996} & Deck & 0.600 & 0.568 & 0.032 \\
& Superstructure & 0.600 & 0.567 & 0.033 \\
& Substructure & 0.600 & 0.569 & 0.031 \\
& Deck & 0.600 & 0.590 & 0.010 \\
1998 & Superstructure & 0.600 & 0.593 & 0.007 \\
& Substructure & 0.600 & 0.591 & 0.009 \\
& Deck & 0.600 & 0.665 & 0.065 \\
2000 & Superstructure & 0.600 & 0.666 & 0.066 \\
& Substructure & 0.600 & 0.669 & 0.069 \\
& Deck & 0.600 & 0.590 & 0.010 \\
2002 & Superstructure & 0.600 & 0.595 & 0.005 \\
& Substructure & 0.600 & 0.591 & 0.009 \\
& Deck & 0.600 & 0.555 & 0.045 \\
2004 & Superstructure & 0.600 & 0.556 & 0.044 \\
& Substructure & 0.600 & 0.557 & 0.043 \\
\hline \hline Mean & Deck & & 3.20 & \\
Errors & Superstructure & & 3.10 & \\
$(\%)$ & Substructure & & 3.20 & \\
\hline
\end{tabular}


Table 4. Conversion from BPM results to BMS input format (Year 1982 of Element \#234 for Bridge \#0312xxx1)

\begin{tabular}{|c|c|c|c|c|c|c|}
\hline & $\begin{array}{c}\text { Condition } \\
\text { State }\end{array}$ & $\begin{array}{l}\text { Prediction } \\
\text { results }\end{array}$ & $\begin{array}{c}\text { Total } \\
\text { elements }\end{array}$ & $\begin{array}{l}\text { Average } \\
\text { proportion } \\
\text { of element } \\
\text { from } 1996 \\
\text { to } 2004\end{array}$ & $\begin{array}{l}\text { Number } \\
\text { of } \\
\text { elements }\end{array}$ & $\begin{array}{c}\text { Number } \\
\text { of } \\
\text { elements } \\
\text { for a BMS }\end{array}$ \\
\hline \multirow{5}{*}{$\begin{array}{c}\text { Proportion } \\
1\end{array}$} & CS1 & $98.48 \%$ & 350 & \multirow{5}{*}{$80.0 \%$} & 275.76 & 276 \\
\hline & CS2 & $1.52 \%$ & 350 & & 4.24 & 4 \\
\hline & CS3 & $0.00 \%$ & 350 & & 0.00 & 0 \\
\hline & CS4 & $0.00 \%$ & 350 & & 0.00 & 0 \\
\hline & CS5 & $0.00 \%$ & 350 & & 0.00 & 0 \\
\hline \multirow{5}{*}{$\begin{array}{c}\text { Proportion } \\
2\end{array}$} & CS1 & $13.64 \%$ & 350 & \multirow{5}{*}{$16.2 \%$} & 7.73 & 8 \\
\hline & $\mathrm{CS} 2$ & $86.36 \%$ & 350 & & 48.97 & 49 \\
\hline & $\mathrm{CS} 3$ & $0.00 \%$ & 350 & & 0.00 & 0 \\
\hline & CS4 & $0.00 \%$ & 350 & & 0.00 & 0 \\
\hline & CS5 & $0.00 \%$ & 350 & & 0.00 & 0 \\
\hline \multirow{5}{*}{$\begin{array}{c}\text { Proportion } \\
3\end{array}$} & CS1 & $1.52 \%$ & 350 & \multirow{5}{*}{$3.8 \%$} & 0.20 & 0 \\
\hline & $\mathrm{CS} 2$ & $87.88 \%$ & 350 & & 11.69 & 12 \\
\hline & $\mathrm{CS} 3$ & $10.61 \%$ & 350 & & 1.41 & 1 \\
\hline & CS4 & $0.00 \%$ & 350 & & 0.00 & 0 \\
\hline & CS5 & $0.00 \%$ & 350 & & 0.00 & 0 \\
\hline \multicolumn{5}{|c|}{ Total number of elements } & 350.00 & 350 \\
\hline
\end{tabular}


Table 5. Results of BPM for BMS inputs

\begin{tabular}{|c|c|c|c|c|c|c|c|c|}
\hline Year & $\begin{array}{r}1968 \\
(\%) \\
\end{array}$ & $\begin{array}{r}1970 \\
(\%) \\
\end{array}$ & & $\begin{array}{r}1972 \\
(\%) \\
\end{array}$ & $\begin{array}{r}1974 \\
(\%) \\
\end{array}$ & $\begin{array}{r}1976 \\
(\%) \\
\end{array}$ & $\begin{array}{r}1978 \\
(\%) \\
\end{array}$ & $\begin{array}{r}1980 \\
(\%) \\
\end{array}$ \\
\hline CS1 & 91.10 & 94.31 & & 80.75 & 79.52 & 80.51 & 92.76 & 91.35 \\
\hline $\mathrm{CS} 2$ & 8.90 & 5.69 & & 17.46 & 17.42 & 18.92 & 7.24 & 8.65 \\
\hline $\mathrm{CS} 3$ & 0.00 & 0.00 & & 1.78 & 3.05 & 0.58 & 0.00 & 0.00 \\
\hline CS4 & 0.00 & 0.00 & & 0.00 & 0.00 & 0.00 & 0.00 & 0.00 \\
\hline CS5 & 0.00 & 0.00 & & 0.00 & 0.00 & 0.00 & 0.00 & 0.00 \\
\hline Total & 100.00 & 100.00 & & 100.00 & 100.00 & 100.00 & 100.00 & 100.00 \\
\hline Year & $\begin{array}{r}1982 \\
(\%) \\
\end{array}$ & $\begin{array}{r}1984 \\
(\%) \\
\end{array}$ & $\begin{array}{r}1986 \\
(\%) \\
\end{array}$ & $\begin{array}{r}1988 \\
(\%) \\
\end{array}$ & $\begin{array}{r}1990 \\
(\%) \\
\end{array}$ & $\begin{array}{r}1992 \\
(\%) \\
\end{array}$ & $\begin{array}{r}1994 \\
(\%) \\
\end{array}$ & $\begin{array}{r}\text { Average } \\
(\%) \\
\end{array}$ \\
\hline CS1 & 81.05 & 78.79 & 80.00 & 88.65 & 80.00 & 80.00 & 80.00 & 84.20 \\
\hline $\mathrm{CS} 2$ & 18.54 & 17.47 & 17.75 & 11.35 & 16.20 & 16.20 & 16.49 & 14.16 \\
\hline CS3 & 0.40 & 3.68 & 2.25 & 0.00 & 3.63 & 3.80 & 3.51 & 1.62 \\
\hline CS4 & 0.00 & 0.06 & 0.00 & 0.00 & 0.17 & 0.00 & 0.00 & 0.02 \\
\hline CS5 & 0.00 & 0.00 & 0.00 & 0.00 & 0.00 & 0.00 & 0.00 & 0.00 \\
\hline Total & 100.00 & 100.00 & 100.00 & 100.00 & 100.00 & 100.00 & 100.00 & 100.00 \\
\hline
\end{tabular}

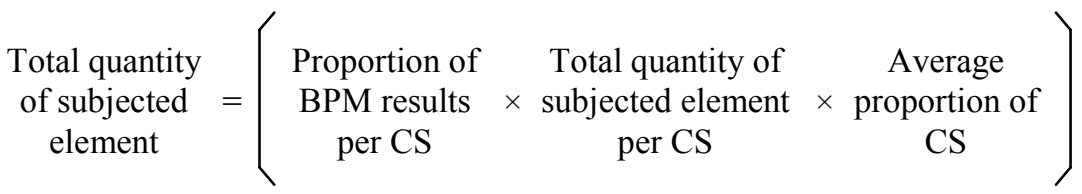

\section{[Equation 1]}


Table 6. Prediction errors of the BPM using forward comparisons

\begin{tabular}{|c|c|c|c|c|c|c|c|}
\hline & & CS1 & CS2 & $\mathrm{CS} 3$ & CS4 & CS5 & Total \\
\hline \multirow{4}{*}{1996} & Results & $84.20 \%$ & $14.18 \%$ & $0.10 \%$ & $1.52 \%$ & $0.00 \%$ & $100.00 \%$ \\
\hline & Existing data & $80.00 \%$ & $14.29 \%$ & $5.71 \%$ & $0.00 \%$ & $0.00 \%$ & $100.00 \%$ \\
\hline & Error & $4.20 \%$ & $0.11 \%$ & $5.62 \%$ & $1.52 \%$ & $0.00 \%$ & \\
\hline & Average error & & & $2.29 \%$ & & & \\
\hline \multirow{4}{*}{1998} & Results & $84.41 \%$ & $13.98 \%$ & $1.58 \%$ & $0.02 \%$ & $0.00 \%$ & $100.00 \%$ \\
\hline & Existing data & $80.00 \%$ & $14.29 \%$ & $5.71 \%$ & $0.00 \%$ & $0.00 \%$ & $100.00 \%$ \\
\hline & Error & $4.41 \%$ & $0.31 \%$ & $4.13 \%$ & $0.02 \%$ & $0.00 \%$ & \\
\hline & Average error & & & $1.78 \%$ & & & \\
\hline \multirow{4}{*}{2000} & Results & $87.65 \%$ & $10.76 \%$ & $0.20 \%$ & $1.40 \%$ & $0.00 \%$ & $100.00 \%$ \\
\hline & Existing data & $80.00 \%$ & $14.29 \%$ & $5.71 \%$ & $0.00 \%$ & $0.00 \%$ & $100.00 \%$ \\
\hline & Error & $7.65 \%$ & $3.53 \%$ & $5.52 \%$ & $1.40 \%$ & $0.00 \%$ & \\
\hline & Average error & & & $3.62 \%$ & & & \\
\hline \multirow{4}{*}{2002} & Results & $91.29 \%$ & $6.96 \%$ & $1.20 \%$ & $0.54 \%$ & $0.00 \%$ & $100.00 \%$ \\
\hline & Existing data & $80.00 \%$ & $19.14 \%$ & $0.86 \%$ & $0.00 \%$ & $0.00 \%$ & $100.00 \%$ \\
\hline & Error & $11.29 \%$ & $12.18 \%$ & $0.34 \%$ & $0.54 \%$ & $0.00 \%$ & \\
\hline & Average error & & & $4.87 \%$ & & & \\
\hline \multirow{4}{*}{2004} & Results & $81.86 \%$ & $16.35 \%$ & $0.85 \%$ & $0.93 \%$ & $0.00 \%$ & $100.00 \%$ \\
\hline & Existing data & $80.00 \%$ & $19.14 \%$ & $0.86 \%$ & $0.00 \%$ & $0.00 \%$ & $100.00 \%$ \\
\hline & Error & $1.86 \%$ & $2.79 \%$ & $0.00 \%$ & $0.93 \%$ & $0.00 \%$ & \\
\hline & Average error & & & $1.12 \%$ & & & \\
\hline
\end{tabular}


Table 7 Typical bridge elements tested

\begin{tabular}{llcc}
\hline $\begin{array}{c}\text { Element } \\
\text { Code }\end{array}$ & \multicolumn{1}{c}{ Description } & $\begin{array}{c}\text { No. of } \\
\text { condition states } \\
(\text { CSs })\end{array}$ & Units \\
\hline BELA & Elastomeric Bearing Pad & 3 & ea \\
CDSL & Concrete Deck Slab & 4 & $\mathrm{~m}^{2}$ \\
CPIL & Concrete Pile & 4 & $\mathrm{~m}^{2}$ \\
CPIR & Concrete Pier (excl. any Headstock or Piles) & 4 & $\mathrm{~m}^{2}$ \\
CPRG & Concrete Pre-tensioned Girder & 4 & $\mathrm{~m}^{2}$ \\
JASS & Assembly Joint Seal & 3 & $\mathrm{~m}$ \\
LBGI & Steel(L) Beam / Girder (Load Bearing) & 5 & $\mathrm{~m}^{2}$ \\
MMAS & Brick / Masonry / Reinforced Earth & 3 & $\mathrm{~m}^{2}$ \\
RMET & Metal Railing & 4 & $\mathrm{~m}$ \\
\hline
\end{tabular}


Table 8. Element condition ratings (3CSs) obtained for the case study

\begin{tabular}{|c|c|c|c|c|c|c|c|}
\hline \multirow{2}{*}{$\begin{array}{l}\text { Bridge } \\
\text { number }\end{array}$} & \multirow{2}{*}{$\begin{array}{c}\text { Construction } \\
\text { year }\end{array}$} & \multirow{2}{*}{$\begin{array}{c}\text { Inspection } \\
\text { date } \\
\text { (dd/mm/yyyy) }\end{array}$} & \multirow{2}{*}{$\begin{array}{l}\text { Structure } \\
\text { element type } \\
\text { code }\end{array}$} & \multirow{2}{*}{$\begin{array}{l}\text { Total } \\
\text { element } \\
\text { quantity }\end{array}$} & \multicolumn{3}{|c|}{ Condition State (CS) } \\
\hline & & & & & CS1 & $\mathrm{CS} 2$ & $\mathrm{CS} 3$ \\
\hline \multirow{5}{*}{$5 \times x 0$} & \multirow{5}{*}{1973} & $14 / 09 / 1994$ & \multirow{5}{*}{ BELA } & 80 & 80 & 0 & 0 \\
\hline & & $29 / 11 / 1996$ & & 80 & 80 & 0 & 0 \\
\hline & & $3 / 03 / 1998$ & & 80 & 80 & 0 & 0 \\
\hline & & $26 / 03 / 2003$ & & 80 & 80 & 0 & 0 \\
\hline & & $14 / 12 / 2005$ & & 80 & 80 & 0 & 0 \\
\hline
\end{tabular}


Table 9. BPM results as BMS inputs (Element BELA for Bridge \#5xx0)

\begin{tabular}{cccccc}
\hline \hline Year & $\begin{array}{c}1974 \\
(\%)\end{array}$ & $\begin{array}{c}1976 \\
(\%)\end{array}$ & $\begin{array}{c}1978 \\
(\%)\end{array}$ & $\begin{array}{c}1980 \\
(\%)\end{array}$ & $\begin{array}{c}1982 \\
(\%)\end{array}$ \\
\hline $0.66<\mathrm{CS} 1 \leq 0.99$ & 96.97 & 95.45 & 100.00 & 96.97 & 95.45 \\
$0.33<\mathrm{CS} 2 \leq 0.66$ & 1.52 & 1.52 & 0.00 & 3.03 & 4.55 \\
$0.00 \leq \mathrm{CS} 3 \leq 0.33$ & 1.52 & 3.03 & 0.00 & 0.00 & 0.00 \\
\hline Total & 100.00 & 100.00 & 100.00 & 100.00 & 100.00 \\
\hline \hline Year & $\begin{array}{c}1984 \\
(\%)\end{array}$ & $\begin{array}{c}1986 \\
(\%)\end{array}$ & $\begin{array}{c}1988 \\
(\%)\end{array}$ & $\begin{array}{c}1990 \\
(\%)\end{array}$ & $\begin{array}{c}1992 \\
(\%)\end{array}$ \\
\hline $0.66<\mathrm{CS} 1 \leq 0.99$ & 98.48 & 100.00 & 83.33 & 100.00 & 100.00 \\
$0.33<\mathrm{CS} 2 \leq 0.66$ & 1.52 & 0.00 & 16.67 & 0.00 & 0.00 \\
$0.00 \leq \mathrm{CS} 3 \leq 0.33$ & 0.00 & 0.00 & 0.00 & 0.00 & 0.00 \\
\hline Total & 100.00 & 100.00 & 100.00 & 100.00 & 100.00 \\
\hline
\end{tabular}


Table 10. Comparisons between forward-predictions and existing condition ratings for Bridge \#5xx0

\begin{tabular}{|c|c|c|c|c|c|}
\hline & & CS1 & $\mathrm{CS} 2$ & $\mathrm{CS} 3$ & Total \\
\hline \multirow{4}{*}{1994} & Results & 96.25 & 2.50 & 1.25 & $100.00 \%$ \\
\hline & Existing data & 100.00 & 0.00 & 0.00 & $100.00 \%$ \\
\hline & Error & 3.75 & 2.50 & 1.25 & \\
\hline & Average error & & $50 \%$ & & \\
\hline \multirow{4}{*}{1996} & Results & 97.47 & 2.53 & 0.00 & $100.00 \%$ \\
\hline & Existing data & 100.00 & 0.00 & 0.00 & $100.00 \%$ \\
\hline & Error & 2.53 & 2.53 & 0.00 & \\
\hline & Average error & & $69 \%$ & & \\
\hline \multirow{4}{*}{1998} & Results & 97.47 & 2.53 & 0.00 & $100.00 \%$ \\
\hline & Existing data & 100.00 & 0.00 & 0.00 & $100.00 \%$ \\
\hline & Error & 2.53 & 2.53 & 0.00 & \\
\hline & Average error & & $69 \%$ & & \\
\hline \multirow{4}{*}{2003} & Results & 97.47 & 2.53 & 0.00 & $100.00 \%$ \\
\hline & Existing data & 100.00 & 0.00 & 0.00 & $100.00 \%$ \\
\hline & Error & 2.53 & 2.53 & 0.00 & \\
\hline & Average error & & $69 \%$ & & \\
\hline \multirow{4}{*}{2005} & Results & 96.25 & 2.50 & 1.25 & $100.00 \%$ \\
\hline & Existing data & 100.00 & 0.00 & 0.00 & $100.00 \%$ \\
\hline & Error & 3.75 & 2.50 & 1.25 & \\
\hline & Average error & & $50 \%$ & & \\
\hline
\end{tabular}


Table 11 Average condition rating differences of the 9 typical bridge elements

\begin{tabular}{lcccc}
\hline \hline \multirow{2}{*}{ Description } & $\begin{array}{c}\text { No. of } \\
\text { condition } \\
\text { states (CSs) }\end{array}$ & $\begin{array}{c}\text { Max. error } \\
\text { allowance } \\
(\%)\end{array}$ & \multicolumn{2}{c}{ Prediction difference $(\%)$} \\
\cline { 5 - 5 } & 3 & 33.33 & Min & Max \\
\hline Assembly Joint Seal & 3 & 33.33 & 1.69 & 2.50 \\
Brick / Masonry / Reinforced Earth & 3 & 33.33 & 3.95 & 18.61 \\
Elastomeric Bearing Pad & 4 & 25.00 & 1.14 & 9.52 \\
Concrete-Deck Slab & 4 & 25.00 & 0.71 & 4.18 \\
Concrete-Pile & 4 & 25.00 & 11.03 & 12.21 \\
Concrete-Pier & 4 & 25.00 & 1.63 & 4.47 \\
Concrete-Pre-tensioned Girder & 4 & 25.00 & 0.73 & 1.56 \\
Metal Railing & 5 & 20.00 & 5.81 & 23.89 \\
Steel(L)-Beam / Girder & & & 1.14 & 4.40 \\
\hline
\end{tabular}

